\title{
Handgrip exercise increases postocclusion hyperaemic brachial artery dilatation
}

\author{
S Agewall, G A Whalley, R N Doughty, N Sharpe
}

\begin{abstract}
Objective-To examine the effect of handgrip exercise induced ischaemia on noninvasive assessment of endothelial function in the brachial artery.

Design and setting-High frequency ultrasound was used to measure brachial artery diameter at rest and after reactive hyperaemia induced by forearm cuff occlusion with and without handgrip exercise induced ischaemia.

Subjects -10 healthy subjects, < 40 years, without known cardiovascular risk factors.

Main outcome measures-Brachial artery dilatation and blood flow.

Results-Hyperaemia following forearm occlusion with handgrip exercise induced ischaemia increased brachial artery diameter significantly more than hyperaemia following occlusion alone, 6.9 (3.2)\% and $4.5(1.6) \%$, respectively $(95 \%$ confidence interval $0.3 \%$ to $4.5 \%)$. There was no difference in peak blood flow with and without exercise induced ischaemia Conclusions-Handgrip exercise induced ischaemia with forearm occlusion caused more pronounced brachial artery dilatation than occlusion alone without change in peak blood flow. This suggests continued brachial artery responsiveness to the stimulus of ischaemia despite maximum blood flow and peripheral vasodilatation with occlusion alone.

(Heart 1999;82:93-95)
\end{abstract}

Keywords: forearm blood flow; vasodilatation; handgrip exercise; ischaemia; endothelial function

Department of Medicine, Sahlgrenska University Hospital, Göteborg University, S-413 45 Göteborg, Sweden

S Agewall

Department of Medicine, Faculty of

Medicine and Health

Science, The

University of

Auckland, Auckland, New Zealand

G A Whalley

R N Doughty

N Sharpe

Correspondence to: Dr Agewall

Accepted for publication 28 January 1999
Endothelial dysfunction is a key early event in atherogenesis. ${ }^{1}$ The endothelium regulates vascular tone through the release of vasoactive agents which act on the underlying vascular smooth muscle. Endothelial function is impaired by cigarette smoking, ${ }^{2}$ hypercholesterolaemia, ${ }^{3}$ and in atherosclerosis. ${ }^{4}$ Previous studies have used invasive techniques to evaluate flow mediated arterial dilatation as a measure of endothelial dysfunction. ${ }^{56}$ However, an invasive technique is not suitable for studies of the earliest preclinical stages of atherosclerosis or for repeated assessment after risk factor modification or treatment of disease. Recently a non-invasive method designed to assess endothelial function in systemic arteries, using high resolution external ultrasound imaging, has been developed..$^{2-4}$

The degree of brachial arterial dilatation following forearm occlusion is now commonly used in clinical studies as a measure of endothelial function. Non-invasive measurement of flow mediated dilatation of the brachial artery has been shown to correlate with invasive assessment of coronary artery endothelial function using acetylcholine infusion. ${ }^{6}$

Blood flow in the brachial artery, which is increased following release of forearm cuff occlusion, returns to normal within a few minutes, while the brachial artery dilatation which occurs persists for several minutes after the flow has returned to baseline. ${ }^{7}$ This suggests that the brachial artery dilatation is not entirely a primary flow dependent response. Other factors, such as the stimulus of ischaemia, may cause the more prolonged brachial artery dilatation.

Our aim in this study was to examine the effects of handgrip exercise induced ischaemia during periods of forearm cuff occlusion on postocclusion hyperaemic brachial artery dilatation and flow in healthy subjects.

\section{Methods}

SUBJECTS AND VISITS

Ten adult volunteers were recruited from our institution. All were normotensive, less than 40 years of age, non-smokers, non-diabetic, with normal serum cholesterol, and on no drugs. Each subject underwent six studies of endothelial function in three separate sessions (two studies on each occasion, with 15 minutes' rest between).

During the third of these three sessions, exercise induced ischaemia (handgrip) was induced during the two studies. Altogether four studies without handgrip exercise and two with handgrip exercise (see below) were performed. The mean values from these examinations were used.

\section{PROTOCOL}

After overnight fasting, the subjects were studied resting and supine between 8 am and 10 am. The electrocardiogram was continuously monitored. The subject's left arm was comfortably immobilised in the extended position to allow consistent imaging of the brachial artery. All studies were performed by the same sonographer (SA). Brachial artery diameter was imaged using a $10 \mathrm{MHz}$ compact linear ultrasound transducer (ATL HDI 3000, Bothell, Washington, USA). The brachial artery segment $1-6 \mathrm{~cm}$ above the antecubital crease was located and imaged in the longitudinal plane, ensuring the lumen diameter was maximised and the gain optimised to provide clear arterial wall interfaces. The distance from 
Table 1 Baseline characteristics of subjects

\begin{tabular}{ll}
\hline Age (years) & $31.8(4.3)$ \\
Sex (male/female) & $6 / 4$ \\
Body mass index $\left(\mathrm{kg} / \mathrm{m}^{2}\right)$ & $25.2(2.8)$ \\
Systolic blood pressure $(\mathrm{mm} \mathrm{Hg})$ & $120(8)$ \\
Diastolic blood pressure $(\mathrm{mm} \mathrm{Hg})$ & $80(4)$ \\
Heart rate (beats/min) & $63(6)$ \\
Serum cholesterol $(\mathrm{mmol} / \mathrm{l})$ & $4.4(1.0)$ \\
Plasma glucose $(\mathrm{mmol} / \mathrm{l})$ & $4.7(0.5)$ \\
\hline
\end{tabular}

Values are means (SD).

the antecubital crease was noted and the following examinations were all performed in the same position: $\mathrm{B}$ mode ultrasound images were obtained with gating from the $\mathrm{R}$ wave of the electrocardiogram; arterial flow velocity measurements were obtained using a pulsed Doppler signal at $60^{\circ}$ to the vessel with the range gate $(1.5 \mathrm{~mm})$ in the centre of the artery. Images were recorded on videotape for subsequent offline analysis on the same instrument.

After an initial 15 minute rest period, baseline recordings of brachial artery diameter and flow velocity were performed. A pneumatic cuff, placed around the forearm distal to the imaged brachial artery segment, was inflated to a pressure of $200 \mathrm{~mm} \mathrm{Hg}$ for 4.5 minutes. During the exercise induced ischaemia studies, the subjects were asked to squeeze a rubber stress ball $(10 \times 6 \times 4 \mathrm{~cm})$ slowly and firmly a total of 30 times during the occlusion period. The subjects completed the handgrip exercise during the first two minutes of occlusion. Blood flow was recorded before and immediately after cuff release for 20 seconds. Continuous B mode images were collected for two minutes after cuff release.

DATA ANALYSES

Images were digitally acquired from the videotape and measured in random order by a single observer blinded to the conditions under which the ultrasonic images were obtained. Measurements of the brachial artery diameter were defined as the distance from the leading edge of the near wall to the leading edge of the far wall of the artery along a line perpendicular to the artery's long axis. Baseline brachial artery diameter was calculated as the mean of two measurements of four images. Two measurements were made of three individual images, providing six diameter measurements at 45 and 60 seconds after cuff deflation. The mean diameter of the measurements after 45 and 60 seconds was divided by the average diameter of the rest scan. Diameter changes were expressed as the percentage change relative to the mean baseline scan $(100 \%)$. Volume flow was calculated by multiplying the velocity time integral of the Doppler flow signal for a single pulse wave by the heart rate and

Table 2 Brachial artery vascular reactivity in healthy subjects with and without exercise induced ischaemia (handgrip)

\begin{tabular}{lllc}
\hline & $\begin{array}{l}\text { Without } \\
\text { handgrip }\end{array}$ & With handgrip & p Value \\
\hline Baseline vessel diameter $(\mathrm{mm})$ & $4.4(0.5)$ & $4.5(0.6)$ & $>0.10$ \\
Baseline blood flow (ml/min) & $59(22)$ & $60(32)$ & $>0.10$ \\
Flow mediated dilatation (\%) & $4.5(1.6)$ & $6.9(3.2)$ & 0.032 \\
Peak blood flow (ml/min) & $447(86)$ & $483(172)$ & $>0.10$ \\
\hline
\end{tabular}

Values are means $(\mathrm{SD})$ vessel cross sectional area. Volume flow was measured during rest and the peak response at the maximum flow in a single cardiac cycle during the 10-20 second period after cuff release. The reliability of assessment of brachial artery dilatation was determined from measurement of images recorded on separate days without exercise induced ischaemia, which provided a coefficient of variation of $2.6 \%$ for the same observer.

\section{STATISTICAL METHODS}

Results are presented as mean (SD). Paired $t$ tests were used to compare continuous variables. All test were two sided and a probability (p) value of $<0.05$ was regarded as statistically significant. All statistics were analysed using Statview 4.5 for Macintosh.

\section{Results}

The mean age of the subjects was approximately 32 years; six were male and four female; serum cholesterol and glucose were within normal limits (table 1). The resting baseline vessel diameters and the baseline flows were similar before both types of forearm occlusion, with and without exercise induced ischaemia (table 2).

Following cuff occlusion with exercise induced ischaemia there was a significant increase in brachial artery diameter $(6.9 \%)$ compared with occlusion alone (4.5\%), 95\% confidence interval $0.3 \%$ to $4.5 \%$. There was no difference in peak blood flow with and without exercise induced ischaemia (table 2). Heart rate was unchanged in both groups.

\section{Discussion}

In this study a significant increase in brachial artery diameter was observed following exercise induced ischaemia during the period of forearm cuff occlusion compared with occlusion alone. No significant difference in peak blood flow was observed with the two different approaches, suggesting that brachial artery calibre was influenced by factors other than changes in flow. This finding supports the suggestion from Bhagat et al that the brachial artery dilatation should be called flow associated rather than flow mediated. ${ }^{8}$

Increased blood flow causes dilatation of the vessel locally ${ }^{9}$ and this dilatation can be blocked by an antagonist to endothelium relaxing factor/nitric oxide production by the endothelium. ${ }^{10}$ Thus the dilatation appears dependent on endogenous $\mathrm{NO}$ released in response to increased flow.

A previous study has shown a prolonged duration of brachial artery dilatation following an early peak blood flow, persisting minutes after the blood flow has returned to basal levels. ${ }^{7}$ In the same study, the flow response after one, three, and five minutes of occlusion caused relatively similar blood flow increases after release of the cuff, whereas only five minutes of occlusion resulted in a significant change in artery diameter. The investigators suggested that the brachial artery dilatation, which occurred only following more prolonged occlusion, may be more than a primary flow 
dependent response. A recently published study, also examining the relevance of different occlusion times and using the same technique, reported that the peak blood flow-observed five seconds after cuff deflation-was very similar, with occlusion times of between 1.5 and 8 minutes, whereas a longer cuff occlusion period caused a longer duration of increased flow. ${ }^{11}$ The maximum brachial artery dilatation occurred after 4.5 minutes of occlusion and did not increase with a longer occlusion duration. The investigators suggested that the duration of flow increase was associated with the degree of brachial artery dilatation. However, while the duration of blood flow increase was more pronounced with 8 minutes than with 4.5 minutes of occlusion, brachial artery dilatation was similar after both intervals, indicating that prolonged hyperaemia was not closely associated with further brachial artery dilatation.

Despite more pronounced brachial artery dilatation following exercise induced ischaemia in the present study, we did not find any difference in peak blood flow when the results with and without exercise induced ischaemia were compared. There was a tendency towards a higher peak blood flow with exercise induced ischaemia, but the proportional change in brachial artery calibre following exercise induced ischaemia was much larger. A type II error cannot be excluded and it is possible that if the study group had been larger a significant difference in blood flow might have been found. Another possibility is that the blood flow increase had a longer duration following exercise induced ischaemia, as the blood flow was not measured more than 30 seconds after cuff deflation.

It can also be argued that blood flow estimation was unreliable with the Doppler technique. Small shifts in the angle of the ultrasound beam may influence the results substantially. ${ }^{12}$ In the study, considerable attention was given to maintain a constant position and the angle was fixed to $60^{\circ}$. The results were based on repeated examinations and several measurements, and baseline flows were very consistent.

The underlying mechanism behind the increased brachial artery dilatation with exercise induced ischaemia is uncertain. It is possible that the blood flow, influenced predominantly by the tone of the peripheral arterioles rather than the proximal vessels, may be close to maximum after 4.5 minutes of occlusion and therefore the exercise induced ischaemia cannot further increase the flow, although the proximal brachial artery can still respond to the stimulus of ischaemia. There were no signs of increased sympathetic activity, the subjects tolerated the test well, and heart rate was unchanged during the experiments. The blood pressure cuff was placed around the forearm and it is possible that the handgrip exercise caused a back flow, exposing the brachial artery to an ischaemic stimulus. Forearm muscles proximal to the cuff that were involved in the exercise might have created an ischaemic environment causing the increased brachial artery dilatation.

The increase in brachial artery diameter following forearm occlusion alone is relatively small. ${ }^{2-4}{ }^{11}$ Exercise induced ischaemia with handgrip increases the dilatation and may enhance comparison of different study groups and the effects of intervention, thus potentially reducing the required sample sizes. However, endothelial function is impaired in subjects with atherosclerosis ${ }^{4}$ and it remains to compare subjects with atherosclerotic disease with healthy controls using this technique.

In conclusion we observed that exercise induced ischaemia caused more pronounced brachial artery dilatation than ischaemia without exercise, without significantly affecting the peak blood flow, suggesting that the brachial artery response to occlusion release may be more than a primary flow dependent response.

This study was supported by grants from the Swedish Medical Research Council, Swedish Medical Society. RND is the recipient of the New Zealand National Heart Foundation BNZ Senior Fellowship.

1 Ross R. The pathogenesis of atherosclerosis: a perspective for the 1990s. Nature 1993;362:801-9.

2 Celermajer DS, Sorensen KE, Georgakopoulus D, et al. Cigarette smoking is associated with dose-related and potentially reversible impairment of endotheliumdependent dilation in healthy young adults. Circulation 1993;88:2149-55.

3 Vogel RA, Coretti MC, Plotnick GD. Changes in flowmediated brachial artery vasoactivity with lowering of mediated brachial artery vasoactivity with lowering of Am 7 Cardiol 1996;77:37-40.

4 Lieberman EH, Gerhard MD, Uehata A, et al. Flowinduced vasodilatation of the human brachial artery is impaired in patients $<40$ years of age with coronary artery disease. Am f Cardiol 1996;78:1210-14.

5 Shiode N, Morishima N, Nakayama K, et al. Flow-mediated vasodilation of human epicardial coronary arteries: effect of inhibition of nitric oxide synthesis. F Am Coll Cardiol 1996; 27:304-10.

6 Anderson TJ, Uehata A, Gerhard MD, et al. Close relation of endothelial function in the human coronary and peripheral circulations. F Am Coll Cardiol 1995;26:1235-41.

7 Corretti MC, Plotnick GD, Vogel RA. Technical aspects of evaluating brachial artery vasodilation using highfrequency ultrasound. Am f Physiol 1995;268:H1397-404.

8 Bhagat K, Hingorani A, Vallance P. Flow associated or flow Bhagat K, Hingorani A, Vallance P. Flow associated or flow 1997;78:7-8.

9 Rubanyi GM, Romero C, Vanhoutte PM. Flow-induced release of endothelium-derived relaxing factor. $A m f$ Physiol 1986;250:1115-19.

10 Joannides R, Haefeli WE, Linder L, et al. Nitric oxide is responsible for flow-dependent dilatation of human peripheral conduit arteries in vivo. Circulation 1995;91:1314-19.

11 Leeson P, Thorne S, Donald A, et al. Non-invasive measurements of endothelial function: effect on brachial artery dilatation of graded endothelial dependent and independent stimuli. Heart 1997;78:22-7.

12 Gill RW. Measurements of blood flow by ultrasound: accuracy and sources of error. Ultrasound Med Biol 1985;11: $625-41$. 